\title{
Hypovirus Transmission to Ascospore Progeny by Field-Released Transgenic Hypovirulent Strains of Cryphonectria parasitica
}

\author{
Sandra L. Anagnostakis, Baoshan Chen, Lynn M. Geletka, and Donald L. Nuss
}

First author: The Connecticut Agricultural Experiment Station, P.O. Box 1106, New Haven 06504; second, third, and fourth authors: Center

for Agricultural Biotechnology, University of Maryland Biotechnology Institute, University of Maryland, College Park 20742. Accepted for publication 8 April 1998.

\begin{abstract}
Anagnostakis, S. L., Chen, B., Geletka, L. M., and Nuss, D. L. 1998. Hypovirus transmission to ascospore progeny by field-released transgenic hypovirulent strains of Cryphonectria parasitica. Phytopathology 88:598604.

Strains of the chestnut blight fungus, Cryphonectria parasitica, have been genetically engineered to contain an integrated full-length cDNA copy of the prototypic virulence-attenuating hypovirus CHV1-EP713. Unlike natural hypovirulent $C$. parasitica strains, these transgenic hypovirulent strains are able to transmit virus to ascospore progeny under laboratory conditions. This ability provides the potential to circumvent barriers to cytoplasmic virus transmission imposed by the fungal vegetative incompatibility system. During July 1994, transgenic hypovirulent strains were introduced into a Connecticut forest site (Biotechnology

hypovirus transmission from transgenic hypovirulent strains to ascospore progeny under field conditions. Additionally, it was possible to recover transgenic hypovirulent strains from the test site as long as 2 years after the limited, single-season release. Evidence also was obtained for cytoplasmic transmission of transgenic cDNA-derived hypovirus RNA, including transmission to mycelia of a virulent $C$. parasitica canker after treatment with conidia of a transgenic strain. Finally, a transgenic hypovirulent strain was recovered from a superficial canker formed on an untreated chestnut tree. Genetic characteristics of the recovered strain suggested that the canker was initiated by an ascospore progeny derived from a cross involving an input transgenic hypovirulent strain. The durability of a molecular marker for field-released cDNA-derived hypovirus RNA is discussed.
\end{abstract} Permit 94-010-01). Subsequent analysis of the release site confirmed

The chestnut blight fungus, Cryphonectria parasitica (Murrill) Barr, is thought to have entered the United States from Japan during the late 1800 s on imported Japanese chestnut trees (Castanea crenata) (7). It spread primarily on nursery stock that was sold locally and by mail order. Native American chestnut (Castanea dentata (Marsh.) Borkh.) proved to be very susceptible to infection by this introduced pathogen. Cankers form as C. parasitica grows in and underneath the bark, resulting in destruction of the cambium and eventual girdling of the tree. Within an orchard or forest, $C$. parasitica is spread by organisms that walk or crawl across cankers, picking up inoculum on feet, feathers, and fur (30). In addition, many insects graze on the exposed fungal stromata, and carry inoculum on mouthparts and internally (27). Asexual spores (conidia) may be splash-dispersed, whereas sexual spores (ascospores) are forcibly ejected during autumn and may become airborne (8).

Infection of chestnut blight fungus by viruses of the genus $H y$ povirus (23) results in attenuation of fungal virulence (hypovirulence). Hypovirulence appears to result from hypovirus-mediated alteration of several fungal signal transduction pathways, including those necessary for virulence on chestnut trees $(16,17,20)$. Natural hypovirulence is reported to control chestnut blight in Italy, France, and Switzerland (13,22). When fungal strains are of the same or closely related vegetative compatibility (VC) types, anastomosis allows hypovirus transmission via cytoplasmic mixing $(1,4)$. Thus, virus transmission is predicted to be hampered in

Corresponding author: D. L. Nuss; E-mail address: nuss@ umbi.umd.edu

Publication no. P-1998-0512-01R

(C) 1998 The American Phytopathological Society
Additional keywords: biological control. fungal populations that exhibit a high degree of $\mathrm{VC}$ diversity. Additionally, between 10 and $90 \%$ of colonies derived from conidia formed by natural hypovirulent strains contain virus $(19,28)$, allowing for continual production of virulent inoculum. Hypoviruses are not transmitted to ascospore progeny of a sexual cross involving a natural hypovirulent strain (2). Moreover, the virulent progeny resulting from such crosses can represent a spectrum of VC types due to allelic rearrangement at the genetic loci that govern VC, contributing to increased VC diversity (14). These properties, coupled with a high degree of $\mathrm{VC}$ diversity in native virulent $C$. parasitica populations, are thought to contribute to a reduced level of efficacy of natural hypovirulent strains for biological control of chestnut blight in North American forest ecosystems $(10,11,13,25,26)$.

After cloning and sequence determination for the prototypic hypovirus CHV1-EP713, Choi and Nuss (18) reported the development of a full-length infectious CHV1-EP713 cDNA clone. Transformation of virulent $C$. parasitica strains with this cDNA clone conferred the complete hypovirulence phenotype. Cytoplasmically replicating double-stranded RNA (dsRNA) was launched from the chromosomally integrated cDNA copy, and these strains were able to convert vegetatively compatible virulent strains to hypovirulence. These results established that a hypovirus is indeed the casual agent of hypovirulence and demonstrated the feasibility of engineering hypovirulent fungal strains.

Because transgenic hypovirulent strains contain a chromosomally integrated viral cDNA copy, it was predicted that, unlike natural hypovirulent strains, viral genetic information would be inherited in a Mendelian fashion by the progeny of a sexual cross involving a transgenic hypovirulent strain. This prediction was confirmed in laboratory mating experiments (14); progeny containing the integrated viral cDNA copy also contained the cDNA- 
derived, cytoplasmically replicating, double-stranded form of the viral RNA. Sexual compatibility for $C$. parasitica is controlled by a single mating locus with two alleles, designated MAT-1 and MAT-2 (12). Thus, sexual crossing should allow the transmission of the integrated viral cDNA throughout the virulent fungal population. Additionally, because the progeny represent a spectrum of different VC groups (VCGs), launching of the cytoplasmic viral RNA form in progeny representing new VCGs should result in the potential for expanded vegetative dissemination of the viral genetic information. Stable transmission of virus at a high frequency $(>99 \%)$ to asexual spores of transgenic hypovirulent strains also has been demonstrated in the laboratory (14). Thus, it has been predicted that these combined transmission properties could result in increased dissemination of the hypovirulence phenotype and, consequently, more effective disease management.

After extensive laboratory and greenhouse tests of transgenic hypovirulent $C$. parasitica strains, we were granted a Biotechnology Permit (94-010-01) to conduct a planned release in a forest. This test was initiated in Connecticut during July 1994 to study the transmission of hypoviruses from transgenic hypovirulent strains to ascospore progeny under field conditions and to examine persistence and dissemination of the introduced transgenic strains and cDNA-derived hypovirus RNA.

\section{MATERIALS AND METHODS}

Fungal strains and growth conditions. The study utilized two virus-free virulent $C$. parasitica strains with the following phenotypes. Strain EP154 has mating type allele MAT-2, carries a VCG 39 designation, produces orange pigmentation, and is virulent. Strain EP392 (ATCC 38983) is MAT-1, carries a VCG 39 designation, produces orange pigmentation, and is virulent. Two additional $C$. parasitica strains were used as recipients for transformation with plasmid pXH9, which contains a full-length cDNA copy of the prototypic hypovirus CHV1-EP713, to produce transgenic hypovirulent strains (18). Strain EP155 (ATCC 38755) is mating type $M A T-2$, VCG 40, orange, and virulent, whereas strain EP146 (ATCC 64671) is MAT-1, VCG 9, brown, and virulent. In addition to the CHV1-EP713 full-length cDNA, plasmid pXH9 also contains the Escherichia coli hygromycin B phosphotransferase gene as a selectable marker flanked by the Aspergillus nidulans trp $\mathrm{C}$ promoter and terminator domains. Thus, transgenic strains are also hygromycin resistant $\left(\mathrm{Hyg}^{\mathrm{R}}\right)$ (18). The presence of CHV1EP713 RNA replicating in the cytoplasm of the transgenic strains also results in reduced pigment production. Consequently, the transgenic strains exhibit a white phenotype. The transgenic hypovirulent $C$. parasitica strains are designated by the original virulent strain used as the transformation recipient followed by the transforming plasmid indicated in brackets. For example, 146[pXH9], indicates a transgenic strain generated by transformation of virulent $C$. parasitica strain EP146 with plasmid pXH9. Virulent and transgenic strain stock cultures were maintained on Difco (Detroit, MI) potato dextrose agar (PDA) (24).

Connecticut deployment test site. American chestnut in Connecticut is found in mixed hardwood forests with oak as the dominant canopy tree. Chestnut sprouts often reach a $12 \mathrm{~cm}$ diameter at breast height ( $\mathrm{dbh}$ ) and become mature (capable of flowering) before becoming infected and dying of chestnut blight. During spring 1994, a site was selected in northwestern Connecticut in the Housatonic State Forest in Sharon Township, Litchfield County $\left(41^{\circ} 52^{\prime} 40^{\prime \prime} \mathrm{N}, 73^{\circ} 22^{\prime} 30^{\prime \prime} \mathrm{W}\right.$, elevation $\left.395 \mathrm{~m}\right)$. Trees in the overstory included Quercus coccinea, Acer rubrum, and a Populus sp. Woody plants in the understory included Carya glabra, Q. prinus, A. pennsylvanicum, Hamamelis virginiana, and an Ulmus sp.

Two plots, each $\approx 50 \times 50 \mathrm{~m}$, were selected. In each plot, 12 American chestnut trees that were at least $2.5 \mathrm{~cm}$ dbh and without cankers were chosen as test trees. All additional American chestnut sprouts (at least $2.5 \mathrm{~cm} \mathrm{dbh}$ ) in the plots and throughout the area are being mapped, numbered, and flagged for monitoring as the study continues. To date, 241 additional American chestnut sprout clumps have been mapped in the plots.

Deployment procedures. The 12 test trees in each plot were grouped as 6 pairs. During June 1994, three pairs in each plot were inoculated with virulent $C$. parasitica strain EP154 (MAT-2, VCG 39), and three pairs in each plot were inoculated with virulent strain EP392 (MAT-1, VCG 39). Stems were inoculated by placing a plug of fungal mycelium on PDA into a 10-mm-diameter hole made in the bark with a cork borer and covering with masking tape to retard drying. Each tree received a single inoculation.

The two transgenic hypovirulent strains were deployed either as mycelial plugs (plot 1) or painted conidia (plot 2). Because transgenic strain 146[pXH9] has mating allele MAT-1 and is VCG 9, it is able to mate with virulent strain EP154 (MAT-2) but not with virulent strain EP392 (MAT-1). Additionally, because VCGs 9 and 39 can form transient anastomoses (3), transgenic strain 146[pXH9] can convert both virulent strains by cytoplasmic transfer of hypoviruses. In contrast, the second transgenic strain, 155[pXH9] (MAT-2, VCG 40), is able to mate with virulent strain EP392 (MAT-1) but not with virulent strain EP154 (MAT-2). Because VCGs 39 and 40 are incompatible, this strain is unable to cytoplasmically transmit viruses to either virulent strain.

In both deployment protocols, one canker on one tree of each test pair was treated with a transgenic strain; the canker on the second tree of the pair served as a control. Test cankers inoculated with virulent strain EP154 (MAT-2, VCG 39) were treated with mating compatible, vegetatively compatible transgenic strain 146[pXH9] (MAT-1, VCG 9). Test cankers inoculated with virulent strain EP392 (MAT-1, VCG 39) were treated with mating compatible, vegetatively incompatible transgenic strain 155[pXH9] (MAT-2, VCG 40) (Table 1). For the plug protocol, virulent cankers $\approx 25 \mathrm{~mm}$ in diameter (21 days) were inoculated with one of the two transgenic strains as mycelial plugs on each side and at the top. The rationale underlying the protocol was that conversion of the virulent canker to hypovirulence by anastomosis with a vegetatively compatible transgenic hypovirulent strain would retard the expansion of the canker, reducing the chance of tree death due to girdling. It also was reasoned that the inoculation at the top of the canker would provide a source of conidia to spermatize trichogynes produced by the virulent canker, leading to sexual crossing.

In the second deployment protocol, conidia harvested from laboratory-grown transgenic hypovirulent strains were painted as a concentrated suspension $\left(2 \times 10^{6} / \mathrm{ml}\right.$, in water $)$ directly onto the surface of the virulent test cankers when they were $\approx 35 \mathrm{~mm}$ in diameter (35 days) and again after 70 days. This protocol was designed to test the ability of the transgenic hypovirulent conidia to serve as spermatia in a sexual cross in the field. In this regard, it is noteworthy that female infertility is one of several phenotypic traits that accompany hypovirus-mediated hypovirulence (i.e., hypovirulent strains are typically unable to serve as a female in a sexual cross but are male fertile [5]). A successful mating would be expected to result in one-half of the resulting ascospore progeny containing the integrated viral cDNA and the cDNA-derived cytoplasmically replicating viral RNA and exhibiting $\mathrm{Hyg}^{\mathrm{R}}$. In a separate series of experiments, $C$. parasitica cankers were receptive to spermatization by as early as 17 days after canker initiation and as early in the growing season as May (9). To increase laboratory yields of transgenic conidia for field application, transgenic hypovirulent $C$. parasitica strains were grown under high light intensity as previously described (14). Harvested conidia were filtered through Miracloth (Calbiochem, La Jolla, CA) to remove residual mycelia fragments.

Effect of transgenic hypovirulent strains on canker expansion. Reduced canker expansion rate is one hallmark of anastomosis-mediated conversion of a resident virulent $C$. parasitica strain by hypoviruses from a donor strain. (2). Canker widths were measured at the time of treatment, and measurements were made 
periodically throughout the growing season. Care was taken to sterilize hands and equipment between measurements to prevent accidental spread. This allowed an assessment of the efficiency of hypovirulence conversion of the two virulent strains tested.

TABLE 1. Organization of Housatonic State Forest (Connecticut) test plots for deployment of transgenic hypovirulent Cryphonectria parasitica, canker expansion, and tree condition

\begin{tabular}{|c|c|c|c|c|c|}
\hline \multirow[b]{2}{*}{ Tree } & \multirow{2}{*}{$\begin{array}{l}\text { Test } \\
\text { strain }\end{array}$} & \multirow{2}{*}{$\begin{array}{c}\text { Transgenic } \\
\text { strain }^{\mathrm{a}}\end{array}$} & \multicolumn{2}{|c|}{ Canker area $\left(\mathrm{cm}^{2}\right)$} & \multirow{2}{*}{$\begin{array}{l}\text { Tree condition } \\
\text { Sep. } 1996\end{array}$} \\
\hline & & & Nov. 1994 & Jul. 1995 & \\
\hline \multicolumn{6}{|l|}{ Plot 1} \\
\hline A & 154 & 146[pXH9] & 254.3 & 274.5 & Alive \\
\hline B & 154 & & 510.4 & $1,533.6$ & Dead above canker \\
\hline $\mathrm{C}$ & 392 & 155[pXH9] & 455.9 & 559.6 & Dead above canker \\
\hline $\mathrm{D}$ & 392 & & 478.9 & $1,109.8$ & Dead above canker \\
\hline $\mathrm{E}$ & 154 & 146[pXH9] & 124.6 & 132.7 & Alive \\
\hline $\mathrm{F}$ & 154 & & 245.9 & $1,506.0$ & Dead above canker \\
\hline G & 392 & 155[pXH9] & 213.7 & 642.1 & Dead to ground \\
\hline $\mathrm{H}$ & 392 & & 397.4 & 602.3 & Dead above canker \\
\hline I & 154 & 146[pXH9] & 91.6 & 122.7 & Alive \\
\hline $\mathrm{J}$ & 154 & & 498.5 & $1,994.0$ & Dead above canker \\
\hline $\mathrm{K}$ & 392 & 155[pXH9] & 362.9 & 678.5 & Dead above canker \\
\hline $\mathrm{L}$ & 392 & & 538.9 & $1,306.7$ & Dead above canker \\
\hline \multicolumn{6}{|c|}{ Plot 2} \\
\hline M & 154 & 146[pXH9] & 418.9 & $1,206.3$ & Dead to ground \\
\hline $\mathrm{N}$ & 154 & & 169.6 & $2,769.8$ & Dead to ground \\
\hline $\mathrm{O}$ & 392 & $155[\mathrm{pXH} 9]$ & 298.5 & $1,169.6$ & Dead above canker \\
\hline $\mathrm{P}$ & 392 & & 274.5 & 854.9 & Dead to ground \\
\hline Q & 154 & $146[\mathrm{pXH} 9]$ & 226.9 & 459.7 & Dead above canker \\
\hline $\mathrm{R}$ & 154 & & 329.9 & 769.1 & Dead to ground \\
\hline $\mathrm{S}$ & 392 & $155[\mathrm{pXH} 9]$ & 514.5 & $1,371.6$ & Dead to ground \\
\hline $\mathrm{T}$ & 392 & & 526.6 & $1,451.5$ & Dead to ground \\
\hline $\mathrm{U}$ & 154 & 146[pXH9] & 301.6 & 793.8 & Dead above canker \\
\hline $\mathrm{V}$ & 154 & & 339.6 & 972.6 & Dead above canker \\
\hline W & 392 & 155[pXH9] & 310.9 & $2,221.7$ & Dead to ground \\
\hline$X$ & 392 & & 383.4 & $1,274.9$ & Dead to ground \\
\hline
\end{tabular}

${ }^{a}$ Strains were deployed in plot 1 as mycelial plugs. Strains were deployed in plot 2 as painted conidia. By June 1997, all test trees, except trees A, E, and $\mathrm{O}$, were dead to the ground. Bark was heavily damaged by insects and had been invaded by wood-rotting fungi. Tree A was still alive above the test canker in September 1997, whereas tree O remained alive below the test canker.
Monitoring survival and spread of transgenic strains. Bark samples used to obtain mass isolates of $C$. parasitica from cankers were removed from canker margins with a 2-mm-diameter cork borer inserted into the cambium, placed on $2 \%$ water agar or $2 \%$ water agar containing $0.1 \% 2$-[N-morpholino]-ethanesulfonic acid buffer, pH 5.0 to 5.5 (Sigma Chemical Co., St. Louis), and 0.25\% tannic acid (J. T. Baker Inc., Phillipsburg, NJ), and incubated on a bench top in the laboratory. Plates were examined after 24 and $48 \mathrm{~h}$ with a dissecting microscope at $30 \times$, followed by transferal of fungal colonies to PDA for additional incubation on a bench top. $C$. parasitica colonies subsequently were transferred to PDA and PDA with hygromycin B (Sigma) at $50 \mathrm{mg} /$ liter to test for $\mathrm{Hyg}^{\mathrm{R}}$. Isolates for further testing were grown on cellophane over PDA at $28^{\circ} \mathrm{C}$ for 5 to 7 days, peeled from the cellophane, freeze-dried, and stored frozen.

Mass isolates were not made from inoculated test cankers in either 1994 or 1995 to minimize damage to developing cankers. Bark samples taken during July 1996 from plug-treated cankers were placed cambial side down on agar, whereas bark samples from conidia-treated cankers were placed bark side down on agar. Cankers on remaining living trees were sampled again during June 1997. Treated and untreated trees throughout the test site also were routinely monitored for new cankers. From summer 1994 through autumn 1997, 66 natural cankers were identified and sampled. The sampled trees were all free of cankers when initially located and tagged. The resulting isolates were paired with each other on PDA for VC determination (5), as were other selected isolates derived from trapped spores and perithecia.

The presence of spores in rainwater run-off from tree stems was examined by attaching funnels to the lower trunks of trees within the test site (six untreated chestnut trees and a small hickory tree). The funnels were connected by tubing to large plastic bottles tied to the trees. After each of 10 rain events (from 31 August to 16 October 1995), the bottles were collected, and the rainwater was filtered and plated on water agar containing tannic acid.

Ascospores of $C$. parasitica form and mature within perithecia imbedded in cankers and are discharged into the air during autumn, depending on temperature and moisture conditions. Ascospores were isolated directly from individual perithecia recovered from bark samples from treated and control cankers (5) collected

\section{$\begin{array}{llllllllllllll}1 & 2 & 3 & 4 & 5 & 6 & 7 & 8 & 9 & 10 & 11 & 12 & 13 & 14\end{array}$}

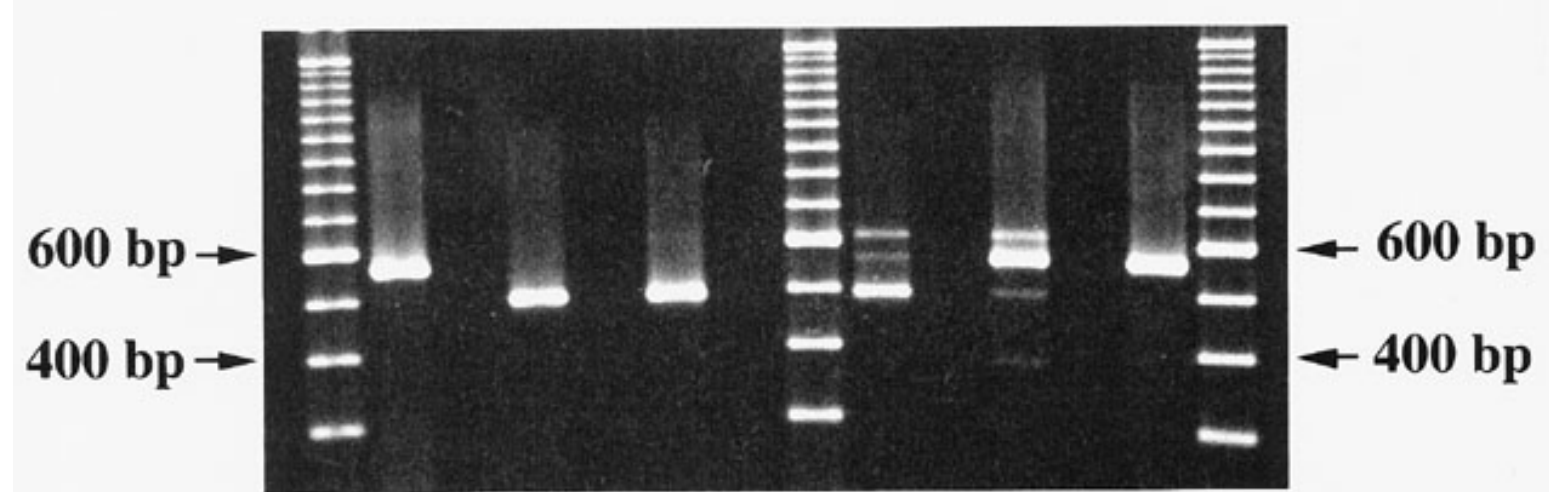

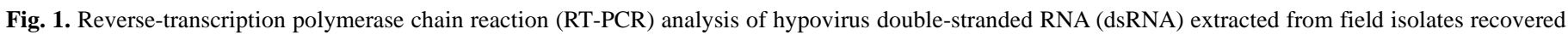

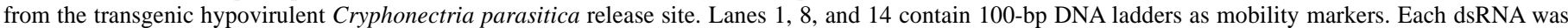

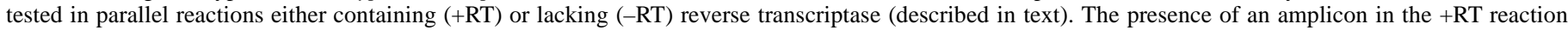

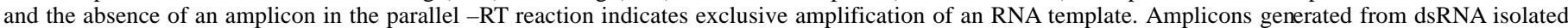

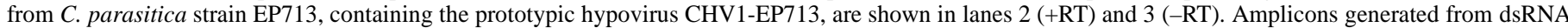

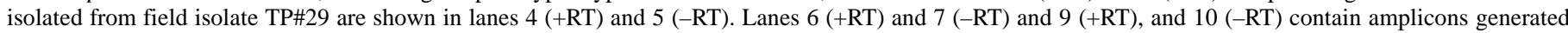

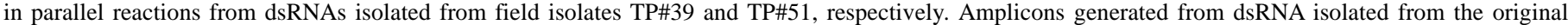

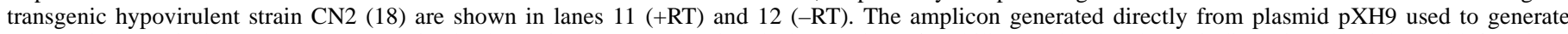

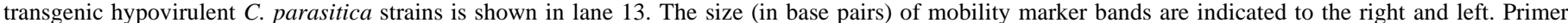

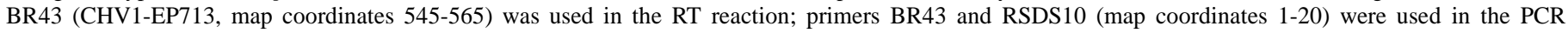

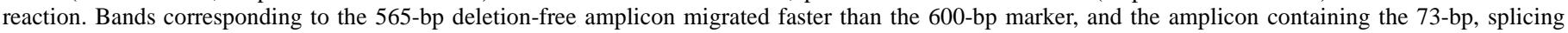
event-mediated deletion migrated just slightly faster than the 500-bp marker. 
25 October 1994, 14 May 1996, and 17 June 1997. Airborne ascospores were collected on two rainy days during early October 1995 and four rainy days during October 1996. A battery-powered Burkard (Rickmansworth, Hertfordshire, England, U.K.) spore trap was placed on a card table under a roof tent erected within the test site, and a fresh plate of tannic acid-water agar was placed in the spore trap every $15 \mathrm{~min}$ for $5 \mathrm{~h}$ each day.

RNA analysis. Hypovirus dsRNA was isolated from mycelia as described by Hillman et al. (24). Viral dsRNAs were subjected to reverse-transcription polymerase chain reaction (RT-PCR) according to the basic protocol described by Shapira et al. (29), under conditions reported by Chen et al. (15). Denaturation of cDNA was performed at $94^{\circ} \mathrm{C}$ for $2 \mathrm{~min}$ in the presence of 0.125 units of AmpliTaq polymerase (Perkin-Elmer, Foster City, CA) per $\mu \mathrm{l}$ and $150 \mathrm{ng}$ each of appropriate oligonucleotide primers in a volume of $20 \mu \mathrm{l}$. Denaturation was followed by 30 cycles of amplification with the following parameters: denaturation for $1 \mathrm{~min}$ at $94^{\circ} \mathrm{C}$, annealing for $1.5 \mathrm{~min}$ at $58^{\circ} \mathrm{C}$, extension for $1.5 \mathrm{~min}$ at $72^{\circ} \mathrm{C}$, and final extension for $10 \mathrm{~min}$ at $72^{\circ} \mathrm{C}$, with the Perkin-Elmer GeneAmp PCR System 9600 thermocycler. Two microliters of each reaction was analyzed by electrophoresis through $2 \%$ NuSieve 3:1 agarose (FMC BioProducts, Rockland, ME) gels. PCR amplicons were isolated from agarose gels by the glasswool cushion method (21) and sequenced with an Applied Biosystems (Foster City, CA) Prism DyeDeoxy Terminator sequencing kit according to the manufacturer's instructions.

\section{RESULTS}

Effect of application of transgenic strains on canker expansion. The results of canker measurements and observations are presented in Table 1. Plug treatment with vegetatively compatible strains (EP154 × 146[pXH9]) retarded canker expansion, which is consistent with conversion of the test canker by hypovirus cytoplasmic transmission. By September 1996, all trees containing virulent control cankers were dead above the test canker or dead to the ground (Table 1). Only trees that were plug treated with compatible transgenic hypovirulent strains were alive above the test canker. Additionally, one conidium-treated tree (tree Q) exhibited a low level of test canker expansion between November 1994 and July 1995. Interestingly, an isolate from the inner bark of this canker contained viral dsRNA but was not $\mathrm{Hyg}^{\mathrm{R}}$. This isolate (TP\#29) was of the same VC type (VCG 39) as the virulent strain used to initiate the canker.

The recovery of a hypovirus RNA-containing, hygromycin sensitive $\left(\mathrm{Hyg}^{\mathrm{S}}\right)$ isolate from a slowly expanding test canker that had been painted with transgenic conidia suggested the possibility that the cDNA-derived hypoviral RNA had been transmitted to the mycelia of the resident virulent test strain, converting it to hypovirulence. Because cDNA-derived CHV1-EP713 dsRNA present in transgenic strains contains a 73-bp deletion within the $5^{\prime}$-noncoding region as a result of a pre-mRNA splicing event during transit from the nucleus to the cytoplasm (15), it was possible to verify by RT-PCR whether the hypovirus RNA isolated from the converted canker originated from the introduced transgenic strain. As shown in Figure 1 (lane 3), the amplicon generated from the 5' portion of the dsRNA present in the field isolate migrated faster than the corresponding amplicon from CHV1-EP713 dsRNA (Fig. 1, lane 2). Sequence analysis confirmed that the former amplicon contained a 73-bp deletion that spanned residues 201 to 273 of the published CHV1-EP713 dsRNA sequence, as previously reported for cDNA-derived hypovirus RNA isolated from laboratory-grown transgenic hypovirulent strain $\mathrm{CN} 2$ (15). Thus, we conclude that cytoplasmic hypoviruses can be transmitted from a transgenic strain to a resident strain comprising a virulent canker even when the transgenic strain is applied as painted conidia.

Transmission of hypoviruses to ascospore progeny. A major goal of this study was to determine whether transgenic hypoviru- lent strains could transmit hypoviruses to ascospore progeny in the field as they do in the laboratory (14). In 1994 (Table 2), ascospore progeny exhibiting $\mathrm{Hyg}^{\mathrm{R}}$ and phenotypic characteristics consistent with hypovirus infection (e.g., white colonies with irregular margins) were recovered from the majority of perithecia. Examination of a random set of isolates revealed the presence of hypovirus dsRNA (data not shown). Pairwise plating studies revealed that 16 VCGs were represented among the recovered ascospore progeny, consistent with the prediction that hypoviruses could be distributed in a spectrum of VCGs as a result of a sexual cross involving a transgenic hypovirulent strain. Ascospores from perithecia on one control canker yielded 59 C. parasitica cultures, none of which were white or $\mathrm{Hyg}^{\mathrm{R}}$. In 1996, a total of 31 perithecia from three treated test trees (trees $\mathrm{G}, \mathrm{K}$, and Q) yielded 1,034 single ascospores, none of which produced white, $\mathrm{Hyg}^{\mathrm{R}}$ colonies. Very few perithecia were observed on the few test trees that survived into 1997, and none contained $\mathrm{Hyg}^{\mathrm{R}}$ ascospore progeny.

Persistence and dissemination of transgenic hypovirulent $\boldsymbol{C}$. parasitica strains. Treated test cankers were not sampled for recovery of input transgenic strains during 1994 or 1995 to minimize damage to developing cankers. Although the condition of many of the treated trees had significantly deteriorated by September 1996 (Table 1), transgenic hypovirulent $C$. parasitica strains (white, $\mathrm{Hyg}^{\mathrm{R}}$ ) were recovered from bark samples taken from a number of treated test cankers during July 1996. These included an inner bark isolate taken from a position adjacent to the plug inoculation site of tree E. Additionally, bark samples taken from test trees in the conidial-spray plot (trees $\mathrm{M}$ and $\mathrm{O}$ ) yielded white, $\mathrm{Hyg}^{\mathrm{R}}$ isolates from the bark surface, even though one of the trees was recently dead from a natural basal canker. During June 1997, white isolates were recovered from bark samples taken from two plug-treated cankers (trees $\mathrm{A}$ and E) and one conidium-painted canker (tree O), but they were not $\mathrm{Hyg}^{\mathrm{R}}$.

Attempts to recover transgenic conidia and ascospores by other methods produced mixed results. Sampling of rainwater collected from seven small untreated trees located within the test site over a 6-week period 1 year after treatment of cankers with transgenic hypovirulent strains (31 August to 16 October 1995) yielded a total of 198 C. parasitica isolates. None of these cultures were $\mathrm{Hyg}^{\mathrm{R}}$ or exhibited phenotypes commonly associated with hypovirus infection.

Ascospores are discharged from perithecia into the air during the autumn, depending on temperature and moisture conditions. Trapping of fungal spores from the air during two rainy days in October 1995 yielded a very large number of diverse fungal isolates, but only 35 isolates were $C$. parasitica. Five (15\%) of the thirty-five isolates were transgenic. Similar trapping attempts during four rainy days in October 1996 yielded 1,183 total fungal isolates that included $115 \mathrm{C}$. parasitica cultures. However, none of these isolates were $\mathrm{Hyg}^{\mathrm{R}}$. Additionally, examination of 15 of these cultures with atypical morphology revealed no hypovirus dsRNA.

TABLE 2. Ascospore progeny recovered from perithecia isolated from test cankers treated with transgenic hypovirulent Cryphonectria parasitica

\begin{tabular}{|c|c|c|c|c|c|c|c|}
\hline \multirow[b]{2}{*}{ Tree } & \multicolumn{2}{|c|}{ Treatment } & \multirow{2}{*}{$\begin{array}{c}\text { No. of } \\
\text { perithecia }\end{array}$} & \multirow[b]{2}{*}{$\mathrm{Hyg}^{\mathrm{R}}$} & \multirow[b]{2}{*}{$\mathrm{Hyg}^{\mathrm{S}}$} & \multirow[b]{2}{*}{ Total } & \multirow[b]{2}{*}{$\% \mathrm{Hyg}^{\mathrm{R}}$} \\
\hline & Plug & $\overline{\text { Conidium }}$ & & & & & \\
\hline \multicolumn{8}{|l|}{1994} \\
\hline A & + & & 7 & 29 & 62 & 91 & 32 \\
\hline I & + & & 1 & 10 & 19 & 29 & 34 \\
\hline $\mathrm{K}$ & + & & 8 & 0 & 73 & 73 & 0 \\
\hline M & & + & 3 & 19 & 40 & 59 & 32 \\
\hline $\mathrm{O}$ & & + & 4 & 11 & 47 & 58 & 19 \\
\hline $\mathrm{S}$ & & + & 28 & 366 & 214 & 580 & 63 \\
\hline $\mathrm{X}$ & & & 4 & 0 & 59 & 59 & 0 \\
\hline \multicolumn{8}{|l|}{1996} \\
\hline $\mathrm{G}, \mathrm{K}, \mathrm{Q}$ & + & +(Q) & 31 & 0 & 1,034 & 1,034 & 0 \\
\hline
\end{tabular}


Dissemination also was tested by sampling new cankers that had arisen during the study. Of 66 isolates from natural cankers, 1 (from treated tree I) contained hypovirus RNA but was not $\mathrm{Hyg}^{\mathrm{R}}$. This isolate (designated TP\#39) was not of the same VC type as either the inoculated test canker above the canker from which it was isolated or the two introduced transgenic strains. RT-PCR analysis of the dsRNA confirmed that it was derived from cDNA (Fig. 1, lane 6). Significantly, a $\mathrm{Hyg}^{\mathrm{R}}$, hypovirus-containing isolate (TP\#51) was recovered from a new canker on an untreated tree (tree $X)$ that was $\approx 1 \mathrm{~m}$ from the nearest treated tree (tree $\mathrm{W}$ ). The presence of cytoplasmic cDNA-derived viral RNA was confirmed by RT-PCR (Fig. 1, lane 9). However, inspection of the gel revealed two amplification products in addition to the amplicon that contained the 73-bp splicing-derived deletion. One of the additional amplicons comigrated with the deletion-free amplicon generated from plasmid pXH9 that contains the full-length $\mathrm{CHV} 1-$ EP713 cDNA sequence. Analysis of this amplicon demonstrated the absence of the 73-bp deletion and confirmed a nucleotide sequence identical to that of the wild-type CHV1-EP713 RNA sequence. This result indicates that some unspliced cDNA-derived hypovirus RNA was translocated to the cytoplasm and initiated the replication cycle. The second extra amplification product (slowest migrating band) was sensitive to $\mathrm{S} 1$ nuclease, indicating that it was single-stranded DNA that arose as a result of asymmetric amplification. Sequence analysis of this product revealed a mixture of amplified DNAs corresponding to both the deletion-containing and deletion-free copies of the CHV1-EP713 noncoding strand.

When isolate TP\#51 was paired with a set of VC tester strains, it was unique among isolates from the plots and was not VCG 39, as were both of the virulent test strains, EP154 and EP392, nor was it VCG 9 or 40, as were the introduced transgenic strains, 146[pXH9] and 155[pXH9]. The combined characteristics are consistent with the possibility that the new superficial canker was initiated by an ascospore progeny derived from a mating event involving a transgenic hypovirulent strain.

Further analysis of the viral RNAs present in the $\mathrm{Hyg}^{\mathrm{S}}$ mass isolates recovered from surviving treated trees (trees A, E, and O) and additional natural cankers (trees 41 and 241) in early 1997 also revealed the presence of both deletion-free and deletion-containing amplicons (data not shown). Because there are no indications that CHV1-EP713-related (European origin) hypoviruses had been introduced into the general test site area previously, it is likely that the deletion-free as well as the deletion-containing hypovirus RNAs present in this collection of hypovirulent mass isolates are cDNA-derived hypoviruses. These results are consistent with the proposal that cDNA-derived viral RNA appears to persist to a greater extent than input transgenic fungal strains.

Interestingly, isolates from 54 of the 66 natural cankers have been separated into 32 VCGs, and a total of 61 VCGs have been identified among all of the isolates (including ascospore isolates) recovered from the plot to date. A total of 445 of the isolates from the plot, so far, have been placed in our stock collection. This collection will serve as a basis for examining the genetic diversity of the pathogen in this forest area over time, after the limited release of transgenic hypovirulent stains $(10,13)$.

\section{DISCUSSION}

The protocols employed in this study for the single-season, limited release of transgenic hypovirulent $C$. parasitica were designed to test three primary issues: transmission of hypoviruses to ascospore progeny under field conditions, persistence in the ecosystem, and dissemination. Biological control was not a primary issue in this study, because single introductions of hypovirulent $C$. parasitica strains previously have not resulted in establishment of biological control in North American forest ecosystems (6).

The transgenic hypovirulent strains behaved as nontransgenic hypovirulent strains in terms of reducing expansion of virulent cankers when delivered as plug treatments (i.e., transgenic hypovirulent strains that were similar in VC to the test virulent canker were able to convert the resident virulent strain to hypovirulence and retard canker expansion [3] [Table 1]). Plug treatment with vegetatively incompatible transgenic hypovirulent strains had no significant effect on test canker expansion. The availability of a molecular marker for cDNA-derived hypovirus RNA, a 73-bp splicing event-mediated deletion within the 5 '-noncoding region, provided the means with which to demonstrate by RT-PCR that a test canker also could be converted by hypovirus RNA delivered as painted conidia (Fig. 1, lane 4, TP\#29). This observation has implications for efforts to optimize the mode of application of both natural and transgenic hypovirulent strains for effective biological control. A measurement of the efficiency of virulent canker conversion as a function of the frequency and intensity of transgenic conidial deployment should be a priority in future studies.

It is clear from the results presented in Table 2 that hypoviruses can be readily transmitted from transgenic hypovirulent strains to ascospore progeny in the field, as was previously demonstrated in the laboratory (14). This property contrasts with the consistent failure of hypoviruses to be transmitted from nontransgenic hypovirulent strains to ascospore progeny under a variety of artificial and natural conditions (5) and is one of the primary bases for predicting enhanced biocontrol potential for transgenic hypovirulent strains. However, inspection of Table 2 also reveals that for perithecia isolated from several trees only $\approx 30 \%$ of the ascospore progeny contained integrated viral cDNA and exhibited $\mathrm{Hyg}^{\mathrm{R}}$. This value differs from the $50 \%$ inheritance frequency observed for ascospore progeny of laboratory crosses involving transgenic strain 155[pXH9] (14). The inheritance frequency for integrated hypovirus cDNA in field crosses clearly merits further detailed examination.

As indicated in Table 2, transgenic ascospore progeny were readily recovered from perithecia taken from both plug-treated and conidiapainted test cankers during October 1994, just months after treatment of test cankers with transgenic hypovirulent strains. Cankers were not sampled in 1995, to minimize damage to developing cankers. Interestingly, subsequent sampling of perithecia taken from treated test cankers in 1996 and 1997 failed to recover any transgenic ascospore progeny (Table 2). This result suggests that repeated introduction of transgenic hypovirulent conidia is required to produce transgenic ascospore progeny year after year. In the single-season release protocol used in this study, potential sources of transgenic conidia are limited within the test site due to hypovirus-mediated suppression of conidia production by persisting transgenic hypovirulent strains, death of inoculated stems, and invasion of test cankers by wood-rotting fungi.

Although there is no immediately apparent rationale for expecting transgenic hypovirulent $C$. parasitica strains to be any less ecologically fit than natural hypovirulent strains, it was of interest to determine how long after a limited, single-season release one could recover transgenic mycelia from the test site. Transgenic hypovirulent strains were readily recoverable from treated cankers that survived through the summer of 1996, 2 years after transgenic strain introduction. However, as was the case for transgenic ascospore progeny, recovery of transgenic mass isolates also was hampered by increasing death of inoculated stems from newly developed natural cankers and virulent test cankers, physically reducing sources for direct recovery of transgenic strains. Transgenic strains were never recovered from collected stem rainwater run-off, whereas recovery of transgenic spores from the air was more successful. Consistent with the reduced number of surviving treated cankers, the rate of recovery of transgenic spores from the air also declined with time after initial release.

The presence of molecular markers in both fungal $\left(\mathrm{Hyg}^{\mathrm{R}}\right)$ and viral (73-bp deletion) genomes greatly facilitated the ability to monitor the independent dissemination of the input transgenic hypovirulent fungal strains and cDNA-derived hypovirus RNA. In 
addition to the example of isolate TP\#29 discussed above, isolate TP\#39, obtained from a new superficial canker located on the lower portion of a treated tree, also contained cDNA-derived hypovirus RNA (Fig. 1, lane 6) and was Hyg'. This canker developed below a test canker that had been plug treated with a transgenic strain but was not of the same VC type as either the virulent test strain or the introduced transgenic strain. Consequently, it is unlikely to have been started by conidia or mycelial fragments from either of these sources but may represent a natural canker that was subsequently converted by a propagule from the converted test canker above. Most interesting was the recovery of isolate TP\#51 from a new superficial canker on an untreated tree. This isolate was shown by RT-PCR to contain a mixture of both deletion-free CHV1-EP713 dsRNA and viral dsRNA containing the splicing-mediated 73-bp deletion characteristic of cDNA-derived viral dsRNA (Fig. 1, lane 9). Hyg $^{\mathrm{R}}$ and the presence of cDNAderived viral dsRNA coupled with the fact that the isolate was a unique VC type, rather than the VC types of the input $C$. parasitica strains, are properties consistent with the possibility that this superficial canker was initiated by a transgenic ascospore. Interestingly, no examples of $\mathrm{Hyg}^{\mathrm{R}}$, virus-free isolates were recovered in this study.

The presence of both deletion-containing and deletion-free amplicons in the RT-PCR reaction for isolate TP\#51 (Fig. 1, lane 9) was unexpected. One explanation for this observation is that a portion of the cDNA-derived viral RNA that is continually being produced in the nuclei of transgenic hypovirulent strains completely escaped splicing events during transport to the cytoplasm and was followed by initiation of the viral RNA replication cycle. This deletion-free viral RNA might be expected to favorably compete with the deleted form during replication over time. This scenario is consistent with the recent observation that after extensive passage in the laboratory, the original transgenic hypovirulent strain, CN2 (18), contained deletion-free viral dsRNA, now as the major component (Fig. 1 lane 11). Thus, it appears that the splicing-mediated 73-bp deletion found in the $5^{\prime}$-noncoding region, while a valid molecular marker for cDNA-derived viral RNA, is not a durable field marker. We suggest alternative molecular markers for the viral genome in future field releases, such as the introduction of a NotI linker at the unique SnaBI site within the 3 '-noncoding region at CHV1-EP713 map position 12,038, as previously reported by Choi and Nuss (18) for transgenic hypovirulent strain 155 [pXH103].

The results of this study clearly indicate that although transgenic hypovirulent strains persist in the ecosystem for at least 2 years after a limited single-season release, the input fungal load is not maintained and drops precipitously during the first 2 years. This reduction is partially due to death and decay of treated test cankers. These characteristics are similar to those observed for field-released natural hypovirulent strains (6). On the positive side, transmission of hypoviruses from transgenic hypovirulent strains to ascospore progeny was readily observed under the field conditions in this limited, single-season release protocol. Production of transgenic ascospore progeny appeared to depend on a continued supply of transgenic conidia. Evidence also was obtained for transmission of cDNA-derived viral dsRNA independent of the transgenic host mycelia to virulent strains, resulting in conversion to hypovirulence and even possible initiation of a new superficial canker by transgenic ascospore progeny. The fact that such events were detected at all among the relatively low number of field isolates examined during the course of this study suggest that such events may have occurred at a reasonably high frequency within the test site.

The generally positive results obtained in this limited field release provide a firm basis for extending field studies of transgenic hypovirulent $C$. parasitica strains. Our next step will be an intensive release of transgenic strains in a small area over several years. Using the basic information reported here, we will attempt a population replacement strategy, replacing indigenous, virulent C. parasitica with nonlethal, transgenic counterparts in an area with an abundance of native American chestnut sprouts. This will allow us to assess the value of these new strains for biological control of chestnut blight.

\section{ACKNOWLEDGMENTS}

We thank P. Sletten for excellent technical assistance. This work was supported by NRICGP grant 95-37312-1638, McIntire-Stennis funds, a Federal FOCUS grant, State of Connecticut funds provided to the Connecticut Agricultural Experiment Station, and the University of Maryland Biotechnology Institute (to D. L. Nuss).

\section{LITERATURE CITED}

1. Anagnostakis, S. L. 1977. Vegetative incompatibility in Endothia parasitica. Exp. Mycol. 1:306-316.

2. Anagnostakis, S. L. 1982. Biological control of chestnut blight. Science 215:466-471.

3. Anagnostakis, S. L. 1983. Conversion to curative morphology in Endothia parasitica and its restriction by vegetative compatibility. Mycologia 75:777-780.

4. Anagnostakis, S. L. 1984. The mycelial biology of Endothia parasitica II. Vegetative incompatibility. Pages 499-507 in: The Ecology and Physiology of the Fungal Mycelium. D. H. Jennings and A. D. M. Rayner, eds. Cambridge University Press, Cambridge.

5. Anagnostakis, S. L. 1988. Cryphonectria parasitica, cause of chestnut blight. Pages 123-136 in: Advances in Plant Pathology. G. S. Sidhu, ed. Academic Press, London.

6. Anagnostakis, S. L. 1990. Improved chestnut tree condition maintained in two Connecticut plots after treatments with hypovirulent strains of the chestnut blight fungus. For. Sci. 36:113-124.

7. Anagnostakis, S. L. 1994. Chestnuts and introduction of chestnut blight. Annu. Rep. North. Nut Growers Assoc. 83:39-42.

8. Anagnostakis, S. L. 1995. The pathogens and pests of chestnuts. Pages 125-145 in: Advances in Botanical Research, vol. 21. J. H. Andrews and I. C. Tommerup, eds. Academic Press, London.

9. Anagnostakis, S. L. Introduction of new genotypes into ascospore progeny of Cryphonectria parasitica in the field. Mycol. Res. In press.

10. Anagnostakis, S. L., Hau, B., and Kranz, J. 1986. Diversity of vegetative compatibility groups of Cryphonectria parasitica in Connecticut and Europe. Plant Dis. 70:536-538.

11. Anagnostakis, S. L., and Kranz, J. 1987. Population dynamics of Cryphonectria parasitica in a mixed-hardwood forest in Connecticut. Phytopathology 77:751-754.

12. Arie, T., Christiansen, S. K., Yoder, O. C., and Turgeon, B. G. 1997. Efficient cloning of ascomycete mating type genes by PCR amplification of the conserved MAT HMG box. Fungal Genet. Biol. 21:118-130.

13. Bissegger, M., Rigling, D., and Heiniger, U. 1997. Population structure and disease development of Cryphonectria parasitica in European chestnut forests in the presence of natural hypovirulence. Phytopathology 87: 50-59.

14. Chen, B., Choi, G. H., and Nuss, D. L. 1993. Mitotic stability and nuclear inheritance of integrated viral cDNA in engineering hypovirulent strains of the chestnut blight fungus. EMBO J. 12: 2991-2998.

15. Chen, B., Craven, M. G., Choi, G. H., and Nuss, D. L. 1994. cDNAderived hypovirus RNA in transformed chestnut blight fungus is spliced and trimmed of vector nucleotides. Virology 202:441-448.

16. Chen, B., Gao, S., Choi, G. H., and Nuss, D. L. 1996. Extensive alteration of fungal gene transcript accumulation and elevation of G-proteinregulated cAMP levels by a virulence-attenuating hypovirus. Proc. Natl. Acad. Sci. USA 93:7996-8000.

17. Choi, G. H., Chen, B., and Nuss, D. L. 1995. Virus-mediated or transgenic suppression of a G-protein $\alpha$ subunit and attenuation of fungal virulence. Proc. Natl. Acad. Sci. USA 92:305-309.

18. Choi, G. H., and Nuss, D. L. 1992. Hypovirulence of chestnut blight fungus conferred by an infectious viral cDNA. Science 257:800-803.

19. Enebak, S. A., MacDonald, W. L., and Hillman, B. I. 1994. Effect of dsRNA associated with isolates of Cryphonectria parasitica from the central Appalachians and their relatedness to other dsRNAs from North America and Europe. Phytopathology. 84:528-534.

20. Gao, S., and Nuss, D. L. 1996. Distinct roles for two G protein $\alpha$ subunits in fungal virulence, morphology and reproduction revealed by targeted gene disruption. Proc. Natl. Acad. Sci. USA 93:14122-14127.

21. Heery, D. M., Gannon, F., and Powell, R. 1990. A simple method for subcloning DNA fragments from gel slices. Trends Genet. 6:173.

22. Heiniger, U., and Rigling, D. 1994. Biological Control of Chestnut Blight 
in Europe. Annu. Rev. Phytopathol. 32:581-599.

23. Hillman, B. I., Fulbright, D. W., Nuss, D. L., and Van Alfen, N. K. 1995. Hypoviridae. Pages 261-264 in: Virus Taxonomy. F. A. Murphy, C. M. Fauquet, D. H. L. Bishop, S. A. Ghabrial, A. W. Jarvis, G. P. Martelli, M. A. Mayo, and M. D. Summers, eds. Springer-Verlag, New York.

24. Hillman, B. I., Shapira, R., and Nuss, D. L. 1990. Hypovirulence-associated suppression of host functions in Cryphonectria parasitica can be partially relieved by high light intensity. Phytopathology 80: 950-956.

25. Liu, Y.-C., and Milgroom, M. G. 1996. Correlation between hypovirus transmission and the number of vegetative incompatibility (vic) genes different among isolates from a natural population of Cryphonectria parasitica. Phytopathology 86:79-86.

26. MacDonald, W. L., and Fulbright, D. W. 1991. Biological control of chestnut blight: Use and limitation of transmissible hypovirulence. Plant Dis. 75:651-661.

27. Russin, J. S., Shain, L., and Nordin, G. I. 1984. Insects as carriers of virulent and cytoplasmic hypovirulent isolates of the chestnut blight fungus. J. Econ. Entomol. 77:838-846.

28. Shain, L., and Miller, J. B. 1992. Movement of cytoplasmic hypovirulence agents in chestnut blight cankers. Can. J. Bot. 70:557-561.

29. Shapira, R., Choi, G. H., Hillman, B. I., and Nuss, D. L. 1991. The contribution of defective RNAs to the complexity of viral-encoded doublestranded RNA populations present in hypovirulent strains of the chestnut blight fungus, Cryphonectria parasitica. EMBO J. 10:741-746.

30. Sharf, C. S., and DePalma, N. K. 1981. Birds and mammals as vectors of the chestnut blight fungus (Endothia parasitica). Can. J. Zool. 59:1647-1650. 